\title{
Addenda and corrections to work done on the path-integral approach to classical mechanics
}

\author{
E.Gozzi and M.Regini \\ Dipartimento di Fisica Teorica, Università di Trieste, \\ Strada Costiera 11, P.O.Box 586, Trieste, Italy \\ and INFN, Sezione di Trieste.
}

\begin{abstract}
In this paper we continue the study of the path-integral formulation of classical mechanics and in particular we better clarify, with respect to previous papers, the geometrical meaning of the variables entering this formulation. With respect to the first paper with the same title, we correct here the set of transformations for the auxiliary variables $\lambda_{a}$. We prove that under this new set of transformations the Hamiltonian $\widetilde{\mathcal{H}}$, appearing in our path-integral, is an exact scalar and the same for the Lagrangian. Despite this different transformation, the variables $\lambda_{a}$ maintain the same operatorial meaning as before but on a different functional space. Cleared up this point we then show that the space spanned by the whole set of variables $(\phi, c, \lambda, \bar{c})$ of our path-integral is the cotangent bundle to the reversed-parity tangent bundle of the phase space $\mathcal{M}$ of our system and it is indicated as $T^{\star}(\Pi T \mathcal{M})$. In case the reader feel uneasy with this strange Grassmannian double bundle, we show in this paper that it is possible to build a different path-integral made only of bosonic variables. These turn out to be the coordinates of $T^{\star}\left(T^{\star} \mathcal{M}\right)$ which is the double cotangent bundle of phase-space.
\end{abstract}




\section{Introduction}

In this paper we continue the study of the path-integral formulation of Hamiltonian classical mechanics started in ref. [1] and continued in ref. [2]. Here in particular we provide a better understanding of the geometrical meaning of the space spanned by the variables $\left(\phi^{a}, c^{a}, \lambda_{a}, \bar{c}_{a}\right)$ entering that path-integral [1]. Instead of reviewing here all the previous work, we suggest that the reader not familiar with the formalism read refs. [1, 2] before embarking in this paper.

In refs. [1, 2] we showed that, somehow magically, our path-integral was giving us tools which provided the full Cartan calculus [4] on symplectic spaces but we failed in giving a geometrically clean understanding of the full space we were working on. In those papers there were some incorrect statements regarding the nature of the full space. It took us some time to fully understand which space those variables, $(\phi, c, \lambda, \bar{c})$, were spanning because both $\lambda_{a}$ and $\bar{c}_{a}$ transformed as basis of the vector fields so both of them seemed to span the tangent bundle $T \mathcal{M}$ to the phase-space $\mathcal{M}$ whose coordinates we called $\phi$. Later on things started clearing up .

The first things that got clear was a puzzle we had on the role of the variables $\lambda_{a}$. In ref. [2] we had supposed that the $\lambda_{a}$ transform, under a (symplectic) diffeomorphism of the phase-space variables $\phi^{a}$, as the basis of the vector fields. The transformations are indicated in eqs. (A9) of ref. [2]. But at the same time we knew that the $\lambda_{a}$, under the time evolution, change as indicated in eq.(3.16) of ref. [1]. Now time-evolution is a particular symplectic diffeomorphism so (3.16) of ref. [1] should fall in the class of transformations (A9) of ref. [2]. But this is not the case, as it is easy to see inspecting the two transformations. In (3.16) of ref. [1] there is in fact an extra piece containing the anticommuting variables $c, \bar{c}$ which is not present in (A9) of ref. [2]. Which is the correct transformation? The extra piece present in (3.16) seems to ruin the clean geometrical character of the $\lambda_{a}$ which,we thought, were the basis of the vector fields. We had that belief because in ref. [1] we proved (see eq. (3.32)) that at the operatorial level $\lambda_{a}=-i \frac{\partial}{\partial \phi^{a}}$. Because of that belief we postulated the transformations (A9) of ref. [2].

We prove here in section 2 that the correct transformation (under any diffeomorphism) is the one of ref. [1] with the extra piece made of Grassmannian variables extra piece does not spoil the geometrical meaning of $\lambda$ as the basis of the vector fields. The reason being that one should consider which is the correct space of functions on which the vector fields act as a derivative. A further indication that those of ref. [1] should be the correct transformations comes from the fact that under those transformations the Hamiltonian $\widetilde{\mathcal{H}}$ entering our path-integral is an exact scalar while it was

\footnotetext{
${ }^{1}$ We will see that it is easy to generalize the transformation from the time evolution to any general diffeomorphism.
} 
not so under the other transformation as shown in Appendix A of ref. [2].

Another issue which we have clarified in this paper, and on which we made wrong statements in ref. [1, 2], is the manner to build symmetric tensors. If we use the partial geometrical results already obtained in ref. [1], we can show that any form-valued antisymmetric tensors can be built out of the variables $(\phi, c, \bar{c})$. Motivated by this result we then thought that the symmetric tensors could be built using the $\lambda$ in place of the $\bar{c}$. But it is not so unless we provide a symplectic connection. Details are provided in section 3. Several calculational details are confined in an appendix at the end of the paper.

Cleared up this fact we turned in section 4 to study the geometrical nature of our space. The thing we notice is that the $c^{a}$, together with the $\phi^{a}$, make not really a cotangent bundle $T^{\star} \mathcal{M}$ to phase-space ${ }^{2}$ but a space known in the literature as reversed parity tangent bundle [3] which is indicated as $\Pi T \mathcal{M}$. Putting all this together we show in section 3 that the space spanned by the full set of variables $(\phi, c, \lambda, \bar{c})$ is the cotangent bundle to the reversed-parity tangent bundle of phase-space indicated as $T^{\star}(\Pi T \mathcal{M})$.

The reader may feel a little up uneasy with the strange Grassmannian double bundle we produced with our variables. To lift this uneasiness we provide in section 5 of this paper a purely bosonic path-integral whose $8 \mathrm{n}$-variables are the coordinates not of a Grassmannian bundle but of a more natural $T^{\star}\left(T^{\star} \mathcal{M}\right)$ which is the double cotangent bundle to phase-space.

\section{Correct Transformations of $\lambda$}

As we said in the introduction the main goal of this paper is to clarify the geometrical meaning of the space spanned by the variables $\left(\phi^{a}, c^{a}, \lambda_{a}, \bar{c}_{a}\right)$. The first puzzle we encountered was the following: both $\lambda_{a}$ and $\bar{c}_{a}$ transformed under a (symplectic) diffeomorphism of $\phi$ (which are the coordinates of the phase-space $\mathcal{M}$ ) as the basis of vector fields [1, 2]. For $\lambda$ this was clear from the fact that in the operatorial formulation associated to our path-integral one had $\lambda_{a}=-i \frac{\partial}{\partial \phi^{a}}$, while for $\bar{c}$ we transformed

it that way because [1] $\bar{c}=\frac{\partial}{\partial c^{a}}$ and $c^{a}$ transforms as a form. We thought that it was a little bit strange that we had two copies (one Grassmannian and one commuting) of the basis of vector fields. The first idea was that maybe we had done a mistake in considering $\lambda$ as the basis of vector field. This thought come from considering how the $\lambda_{a}$ change under the simplest of (symplectic) diffeomorphisms that is the Hamiltonian evolution. The Lagrangian associated to our path-integral [1] is:

$$
\widetilde{\mathcal{L}}=\lambda_{a}\left[\dot{\phi}^{a}-\omega^{a b} \partial_{b} H\right]+i \bar{c}_{a}\left[\delta_{b}^{a} \partial_{t}-\omega^{a c} \partial_{c} \partial_{b} H\right] c^{b}
$$

\footnotetext{
${ }^{2}$ We erroneously stated that in refs. [1, 2]
} 
where $H$ is the Hamiltonian? of our system in the phase-space $\mathcal{M}$. From the $\widetilde{\mathcal{L}}$ above we can derive the equation of motion for $\lambda$ :

$$
\partial_{t} \lambda_{a}=-\lambda_{i} \omega^{i j} \partial_{j} \partial_{a} H-i \bar{c}_{i} \omega^{i j} \partial_{j} \partial_{b} \partial_{a} H c^{b}
$$

From (2) one can derive the transformation under an infinitesimal time interval $\Delta t$ :

$$
\lambda_{a}^{\prime}=\left[\delta_{a}^{b}-\omega^{b j} \partial_{j} \partial_{a} H \Delta t\right] \lambda_{b}-i \bar{c}_{i} \omega^{i j}\left(\partial_{j} \partial_{b} \partial_{a} H \Delta t\right) c^{b}
$$

Let us compare this transformation with the one of eq.(A9) of ref. [2] which is the correct one if $\lambda$ were the basis of the vector fields and it is:

$$
\lambda_{a}^{\prime}=\frac{\partial \phi^{b}}{\partial \phi^{\prime a}} \lambda_{b}=\left[\delta_{a}^{b}+\partial_{a}\left(\omega^{b c} \partial_{c} G\right)\right] \lambda_{b}
$$

where $G$ is related to the infinitesimal (symplectic) transformation $\phi^{\prime a}=\phi^{a}-\varepsilon^{a}(\phi)$ as follows: $\varepsilon^{e}=\omega^{e f}\left(\partial_{f} G\right)$. For the infinitesimal time evolution of eq.(3), the $G$ would be $G=-H \Delta t$. In that case eq. (ه) would become

$$
\lambda_{a}^{\prime}=\left[\delta_{a}^{b}-\omega^{b j} \partial_{j} \partial_{a} H \Delta t\right] \lambda_{b}
$$

We see immediately that this is not the correct time-evolution given by eq.(3) the second piece containing $\bar{c}$ and $c$ is totally missing.

The extra piece, under a generic (symplectic) diffeomorphism generated by the $G$ of eq.(14), would turn this equation into:

$$
\lambda_{a}^{\prime}=\lambda_{a}+\omega^{i j}\left(\partial_{j} \partial_{a} G\right) \lambda_{i}+i \bar{c}_{i} \omega^{i j}\left(\partial_{j} \partial_{b} \partial_{a} G\right) c^{b}
$$

Let us first see the effect of this extra piece on the transformation properties of $\widetilde{\mathcal{L}}$ of eq.(11) and on the associated $\widetilde{\mathcal{H}}$ which is

$$
\widetilde{\mathcal{H}}=\lambda_{a} \omega^{a b} \partial_{b} H+i \bar{c}_{a} \partial_{b}\left(\omega^{a c} \partial_{c} H\right) c^{b}
$$

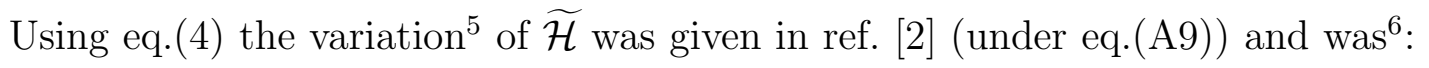

$$
\Delta \widetilde{\mathcal{H}}=\widetilde{\mathcal{H}^{\prime}}\left(\phi^{\prime}\right)-\widetilde{\mathcal{H}}(\phi)=i c^{b} \bar{c}_{a} \omega^{f c}\left(\partial_{c} H\right) \partial_{b} \partial_{f}\left(\omega^{a e} \partial_{e} G\right)+O\left(G^{2}\right)
$$

\footnotetext{
${ }^{3}$ Repeated indices are meant as summed all through this paper

${ }^{4}$ Note that the term with the $c$ and $\bar{c}$ variables has a different sign than in [1]. The correct one is the one written here in this paper.

${ }^{5}$ Of course we change not only $\lambda$ but also the other variables. These ones are changed as in eq. (A9) of ref. [2].

${ }^{6}$ With $\widetilde{\mathcal{H}^{\prime}}\left(\phi^{\prime}\right)$ we indicate an $\widetilde{\mathcal{H}}$ of the same "form" (in the new variables) as the one of eq.(17) but with $H$ replaced by the canonically transformed $H^{\prime}$ indicated in eq.(A9) of ref. [2].
} 
With the new transformation of eq.(6) there will be an extra piece in the variation of $\widetilde{\mathcal{H}}$. This extra piece will come only from the part of $\widetilde{\mathcal{H}}$ containing $\lambda$.It is easy to find this extra variation?:

$$
\Delta_{\text {extra }} \widetilde{\mathcal{H}}=\left(\Delta_{\text {extra }} \lambda_{a}\right) \omega^{a b} \partial_{b} H=i \bar{c}_{i} \omega^{i j}\left(\partial_{j} \partial_{b} \partial_{a} G\right) c^{b} \omega^{a l} \partial_{l} H
$$

Let us now sum the two variations (8) and (9)

$$
\begin{aligned}
\Delta_{\text {tot }} \widetilde{\mathcal{H}} & =\Delta \widetilde{\mathcal{H}}+\Delta_{\text {extra }} \widetilde{\mathcal{H}} \\
& =i c^{b} \bar{c}_{a} \omega^{f c}\left(\partial_{c} H\right) \omega^{a e}\left(\partial_{e} \partial_{b} \partial_{f} G\right)+i \bar{c}_{i} \omega^{i j}\left(\partial_{j} \partial_{b} \partial_{a} G\right) c^{b} \omega^{a l} \partial_{l} H
\end{aligned}
$$

If, in the first piece on the RHS of this equation, we relabel the indeces in the following manner $a \rightarrow i, f \rightarrow a, c \rightarrow l, e \rightarrow j$, and in the second piece we bring the last $c$-variable in front, we get:

$$
\Delta_{t o t} \widetilde{\mathcal{H}}=i c^{b} \bar{c}_{i} \omega^{a l}\left(\partial_{l} H\right) \omega^{i j}\left(\partial_{j} \partial_{b} \partial_{a} G\right)-i c^{b} \bar{c}_{i} \omega^{a l} \partial_{l} H \omega^{i j}\left(\partial_{j} \partial_{b} \partial_{a} G\right)=0
$$

of course this is true up to terms $O\left(G^{2}\right)$ but this does not matter because $\mathrm{G}$ is infinitesimal in an infinitesimal transformation.

So we have proved that the transformations (6) leave the $\widetilde{\mathcal{H}}$ invariant or better $\widetilde{\mathcal{H}}$ behaves as a scalar under a (symplectic) diffeomorphism. To complete the calculations we will now show that the Lagrangian $\widetilde{\mathcal{L}}$ is actually invariant 8 without the need to use the equations of motion?. In appendix A of ref. [2] we proved that under the transformation (4) the $\widetilde{\mathcal{L}}$ changes as

$$
\widetilde{\mathcal{L}}^{\prime}=\widetilde{\mathcal{L}}+i c^{b} \bar{c}_{a} \frac{\partial^{2}\left(\omega^{a c} \partial_{c} G\right)}{\partial \phi^{f} \partial \phi^{b}} \dot{\phi}^{f}-i c^{b} \bar{c}_{a} \frac{\partial^{2}\left(\omega^{a c} \partial_{c} G\right)}{\partial \phi^{f} \partial \phi^{b}} \omega^{f d} \partial_{d} H
$$

The third piece on the RHS of the equation above comes from the term on the RHS of eq. (8). It is the same in fact but with the opposite sign because $\widetilde{\mathcal{L}}$ is related to $-\widetilde{\mathcal{H}}$ under the Legendre transformation. As we know that under the new transformation (6) the $\widetilde{\mathcal{H}}$ will not have that extra piece, the same will happen for $\widetilde{\mathcal{L}}$. We have now only to check if even the second piece on the RHS of eq.(12) will disappear under the new transformation (6). Actually in $\widetilde{\mathcal{L}}^{\prime}$ we have the kinetic piece $\lambda_{a}^{\prime} \dot{\phi}^{\prime a}$ containing $\lambda$ and not present in $\widetilde{\mathcal{H}^{\prime}}$. This will produce, thanks to the new transformation (6), an

\footnotetext{
${ }^{7}$ Of course we keep transforming the other variables $\phi, c, \bar{c}$ as in eq. (A9) of ref. [2]

${ }^{8}$ We keep using the word "invariant" but it is wrong, we mean "scalar". "Invariant" would infact mean $\widetilde{\mathcal{L}}^{\prime}(\phi, \cdots)=\widetilde{\mathcal{L}}(\phi, \cdots)$ while "scalar" means $\widetilde{\mathcal{L}}^{\prime}\left(\phi^{\prime}, \ldots{ }^{(\prime)}\right)=\widetilde{\mathcal{L}}(\phi, \cdots)$. "Invariant" for a Lagrangian would imply there is a conserved quantity under those transformations and, of course, it is not the case here! We are only checking the covariance property of our Lagrangian.

${ }^{9}$ That was a need we had in ref. 22 eq.(A10).
} 
extra term which may cancel the second piece in the RHS of (12). This is in fact so. The extra piece produced is: $i \bar{c}_{i} \omega^{i j}\left(\partial_{j} \partial_{b} \partial_{a} G\right) c^{b} \dot{\phi}^{a}$ which is exactly the opposite of the second piece on the RHS of (12). So this proves that, with the new transformation (6) we obtain, instead of (12), the following:

$$
\widetilde{\mathcal{L}}^{\prime}\left(\phi^{\prime}, \cdots\right)=\widetilde{\mathcal{L}}(\phi, \cdots)
$$

So it seems that the correct transformation for $\lambda$ is (6) which has the extra-piece containing the Grassmannian variables $c, \bar{c}$.

The reader may suspect that the extra piece present in the transformations of $\lambda$ is needed only if we insist in having a new $\widetilde{\mathcal{H}^{\prime}}$ with the same form of the $\widetilde{\mathcal{H}}$ of eq. (17), that means if we insist in having the Lie-derivative of an Hamiltonian flow [1, 2]. We will prove that this is not the case. If we start from the generic Lie-derivative $l_{V}$ of an arbitrary vector-field $V$ (not an Hamiltonian one) it can be written [ 4 ] in a coordinate free way as $l_{V} \equiv d i_{V}+i_{V} d$ where $d$ is the exterior derivative and $i_{V}$ is the interior contraction with the vector field $V$. As $l_{V}$ is a coordinate free expression it must be invariant under any change of coordinates (or diffeomorphism). That Lie-derivative in our notation [1] can be written as:

$$
\widetilde{\mathcal{H}}_{V} \equiv \lambda_{a} V^{a}+i \bar{c}_{a} \partial_{b} V^{a} c^{b}
$$

and एण we will show that in order to have "invariance" of this generic Lie-derivative under any diffeomorphism we need the extra piece in the transformation of $\lambda$.

Let us transform the variables entering entering $\widetilde{\mathcal{H}}_{V}$ as:

$$
\begin{aligned}
\phi^{\prime a} & =\phi^{a}-\varepsilon^{a}(\phi) \\
c^{\prime a} & =c^{a}-c^{b} \partial_{b} \varepsilon^{a} \\
\bar{c}_{a}^{\prime} & =\bar{c}_{a}+\bar{c}_{b} \partial_{a} \varepsilon^{b} \\
\lambda_{a}^{\prime} & =\lambda_{a}+\lambda_{b} \partial_{a} \varepsilon^{b} \\
V^{\prime a} & =V^{a}-V^{b} \partial_{b} \varepsilon^{a}
\end{aligned}
$$

where $\varepsilon^{a}(\phi)$ is a vector field generating a generic diffeomorphism四

\footnotetext{
${ }^{10}$ We see that, if $V^{a}=\omega^{a b} \partial_{b} H$, we end up in our Lie-derivative of the Hamiltonian flow (7). Of course not any $V$ can be written that way, only the Hamiltonian ones.

${ }^{11}$ It is easy to see that if $\varepsilon^{a}=\omega^{a b} \partial_{b} G$ we would end up with our transformations (A9) of ref. [2]. These last transformations are called symplectic diffeomorphisms because they would lead to a new $V^{\prime}$ which could still be written as an Hamiltonian vector field $V^{\prime a}=\omega^{a b} \partial_{b} H^{\prime}$ with only a different $H$. With the calculations shown in the first part of this chapter we can say that (if we use the new transformation for $\lambda$ ) the Lie-derivative of an Hamiltonian vector field is invariant under symplectic diffeomorphism. What we will prove next is that we need that different transformation of $\lambda$ also to prove that a generic Lie-derivative is coordinate free (or invariant) under a generic diffeomorphism.
} 
It is a simple calculation to show that under the transformations above $\widetilde{\mathcal{H}}_{V}$ is not invariant:

$$
\Delta \widetilde{\mathcal{H}}_{V} \equiv \widetilde{\mathcal{H}}_{V^{\prime}}^{\prime}-\widetilde{\mathcal{H}}_{V}=-i \bar{c}_{a} V^{m} \partial_{b} \partial_{m} \varepsilon^{a} c^{b}
$$

If in eq. 18) we transform $\lambda$ with the extra ghost piece as:

$$
\lambda_{a}^{\prime}=\lambda_{a}+\lambda_{b} \partial_{a} \varepsilon^{b}+i \bar{c}_{i}\left(\partial_{a} \partial_{b} \varepsilon^{i}\right) c^{b}
$$

then we get that $\Delta \widetilde{\mathcal{H}}_{V}=0$. So even for a generic Lie-derivative under a generic diffeomorphism we have to change $\lambda$ in a different way in order to have invariance of the Lie-derivative. This proves that this change of $\lambda$ is a fundamental property and it is not related to having treated only the Lie-derivative of Hamiltonian flows under symplectic diffeomorphisms.

We have anyhow now to reconcile what we proved above with the fact that, at the operatorial level, we had got [1]

$$
\lambda_{a}=-i \frac{\partial}{\partial \phi^{a}}
$$

We say that we have to reconcile these two facts because we thought that eq.(22) would imply that the $\lambda_{a}$, being proportional to $\frac{\partial}{\partial \phi^{a}}$, would transform as the basis of vector fields i.e. as indicated in eq.(4) and not as in eq.([6).

The way to reconcile this is the following : $\frac{\partial}{\partial \phi^{a}}$ would transform as a vector field if it were applied to functions only of $\phi$, i.e. $F(\phi)$, but it would transform differently if it were applied to functions of $\phi$ and $c$, i.e. $F(\phi, c)$. We will now show that, if applied to these last functions, they would transform as the $\lambda_{a}$ do in eq.(河).

As explained in appendix A of ref. [2], under the same (symplectic) diffeomorphism which is applied to $\lambda$, the $\phi$ and $c$ transform as:

$$
\begin{aligned}
\phi^{\prime a} & =\phi^{a}-\omega^{a b} \partial_{b} G \\
c^{\prime a} & =\left[\delta_{b}^{a}-\omega^{a c} \partial_{c} \partial_{b} G\right] c^{b}
\end{aligned}
$$

Note that the transformed $c^{\prime}$ depend on $\phi$ via $G$. Let us now take a function of $\phi$ and $c$, i.e. $F(\phi, c)$ and let us transform its arguments.

$$
F(\phi, c)=F\left[\phi\left(\phi^{\prime}\right), c\left(\phi^{\prime}, c^{\prime}\right)\right] \equiv \mathcal{S}\left(\phi^{\prime}, c^{\prime}\right)
$$

If we now apply $\frac{\partial}{\partial \phi^{\prime}}$ on $\mathcal{S}$, we have

$$
\begin{aligned}
\frac{\partial}{\partial \phi^{\prime}} \mathcal{S}\left(\phi^{\prime}, c^{\prime}\right) & =\frac{\partial}{\partial \phi^{\prime}} F\left[\phi\left(\phi^{\prime}\right), c\left(\phi^{\prime}, c^{\prime}\right)\right]= \\
& =\frac{\partial F}{\partial \phi} \frac{\partial \phi}{\partial \phi^{\prime}}+\frac{\partial F}{\partial c} \frac{\partial c}{\partial \phi^{\prime}}
\end{aligned}
$$


Comparing the two RHS of the above equations we can say that:

$$
\frac{\partial}{\partial \phi^{\prime a}}=\frac{\partial \phi^{b}}{\partial \phi^{\prime a}} \frac{\partial}{\partial \phi^{b}}+\frac{\partial c^{b}}{\partial \phi^{\prime a}} \frac{\partial}{\partial c^{b}}
$$

Using the operatorial correspondence described in ref. [1]: $\frac{\partial}{\partial \phi^{\prime a}}=i \lambda_{a}^{\prime}$, $\frac{\partial}{\partial \phi^{a}}=i \lambda_{a}$ and $\frac{\partial}{\partial c^{b}}=\bar{c}_{b}$, we can re-write eq. 28 as

$$
i \lambda_{a}^{\prime}=i \frac{\partial \phi^{b}}{\partial \phi^{\prime a}} \lambda_{b}+\frac{\partial c^{b}}{\partial \phi^{\prime a}} \bar{c}_{b}
$$

One could say that in general $\frac{\partial c^{b}}{\partial \phi^{\prime a}}=0$ because $c$ and $\phi^{\prime}$ are independent coordinates. Actually it is not so because as, we saw in eq.(24), the transformed $c$ depend on $\phi$ and viceversa the original $c$ depends on the transformed $\phi$. So $\frac{\partial c^{b}}{\partial \phi^{\prime a}} \neq 0$.

Let us now proceed to see if eq.(29) is the same as eq.(6). From eqs.(23) and (24) we can derive their inverse which are:

$$
\begin{aligned}
\phi^{a} & =\phi^{a}+\omega^{a b} \partial_{b} G\left(\phi^{\prime}\right) \\
c^{a} & =\left[\delta_{b}^{a}+\omega^{a c} \partial_{c} \partial_{b} G\right] c^{\prime b}
\end{aligned}
$$

and from here we get:

$$
\begin{aligned}
\frac{\partial \phi^{b}}{\partial \phi^{\prime a}} & =\delta_{a}^{b}+\omega^{b i} \partial_{i} \partial_{a} G \\
\frac{\partial c^{b}}{\partial \phi^{\prime a}} & =\omega^{b c} \partial_{c} \partial_{i} \partial_{a} G c^{i}
\end{aligned}
$$

Inserting these expressions into eq.(29) we obtain

$$
\lambda_{a}^{\prime}=\left[\delta_{a}^{b}+\omega^{b i} \partial_{i} \partial_{a} G\right] \lambda_{b}-i\left(\omega^{b c} \partial_{c} \partial_{i} \partial_{a} G\right) c^{i} \bar{c}_{b}
$$

where we have replaced $c^{\prime}$ with $c$ because we keep only terms which are first order in the infinitesimal $G$. Bringing now $\bar{c}$ in front on the second term of the RHS of the equation above we get:

$$
\lambda_{a}^{\prime}=\left[\delta_{a}^{b}+\omega^{b i} \partial_{i} \partial_{a} G\right] \lambda_{b}+i \bar{c}_{b} \omega^{b c} \partial_{c} \partial_{i} \partial_{a} G c^{i}
$$

which is exactly the transformation for $\lambda$ we had in eq. (6). So this proves that operatorially $\lambda_{a}$ act as $\frac{\partial}{\partial \phi^{a}}$ but over the functions $F(\phi, c)$ and this in turn implies that the base-space we should consider first is the one made of $(\phi, c)$. 


\section{$3 \quad$ Symmetric tensors}

Having cleared the issue of the transformations of the $\lambda$ let us now turn to another topic which was only briefly mentioned in refs. [1, 2]. The topic is the following: a generic function of the coordinates $(\phi, c, \bar{c})$, i.e.

$$
\mathcal{F}=\mathcal{F}_{i, j, \cdots n}^{a b \cdots m}(\phi) c^{i} c^{j} \cdots c^{n} \bar{c}_{a} \bar{c}_{b} \cdots \bar{c}_{m}
$$

represent a form-valued anti-symmetric tensor field. This is so because [1] the $c^{a}$ are forms while the $\bar{c}_{a}$ transform as vectors (17). So the strings of $\bar{c}_{a}$ in (36) gives to the $\mathcal{F}$ the character of an anti-symmetric multivector [5] while the strings of $c$ makes it a multi-form. Stripping it of the $c$ variables we get a simple anti-symmetric multivector field:

$$
\mathcal{T}=\mathcal{T}^{a b \cdots m}(\phi) \bar{c}_{a} \bar{c}_{b} \cdots \bar{c}_{m}
$$

Somehow this statement was already present in refs. [1, 2] where forms and tensors were written with our variables $c^{a}, \bar{c}_{a}$ and all the Cartan calculus was given in details.

In those same references [1, 2] we gave hints that symmetric tensors could be built by replacing in (37) the $\bar{c}_{a}$ (which are anticommuting and provide the anti-symmetric character of the tensor $\mathcal{T}$ ) with the variables $\lambda_{a}$ which, being commuting, appeared as the natural objects to build symmetric tensors. We wrongly thought that objects like:

$$
\mathcal{S}=\mathcal{S}^{a b \cdots m}(\phi) \lambda_{a} \lambda_{b} \cdots \lambda_{m}
$$

would transform as symmetric tensors. The reason why this is wrong is because the $\lambda_{a}$ do not transform as indicated in eq.(4), i.e, as vectors, which would make the $\mathcal{S}$ a symmetric multivector, but they transform as indicated in eq. (6). So, as the $\lambda_{a}$ are not anymore vectors, the $\mathcal{S}$ are not anymore symmetric multivectors. The same would happen if we use the operatorial representation of $\lambda$ i.e. $\lambda_{a}=-i \frac{\partial}{\partial \phi^{a}}$. For example, let us build a symmetric object with two indices which would be: $\mathcal{S}^{a b} \frac{\partial}{\partial \phi^{a}} \frac{\partial}{\partial \phi^{b}}$ It is well known that, while a single derivative $\frac{\partial}{\partial \phi^{a}}$ would transform as a vector component, a double derivative $\frac{\partial}{\partial \phi^{a}} \frac{\partial}{\partial \phi^{b}}$ would not transform as the product of two vector components, unless we introduce a connection [6].

So the problem is to check if in our formalism we can find a manner to build not only anti-symmetric but also symmetric tensors and eventually even mixed ones. For the moment let us focus our attention on the symmetric ones.

We see that without a connection there is no manner to make symmetric even a 2-tensor. So the new crucial ingredient to pull in our construction seems to be a connection $\Gamma$. A connection is an object needed to do the parallel transport of vectors [6]. Under a diffeomorphism $\phi^{\prime a}=\phi^{\prime a}(\phi)$ of the space $\mathcal{M}$, the connection does not 
transform as a tensor but in the following manner:

$$
\Gamma_{c b}^{\prime a}=\frac{\partial \phi^{r}}{\partial \phi^{\prime c}}\left(\frac{\partial \phi^{\prime a}}{\partial \phi^{l}} \Gamma_{r m}^{l} \frac{\partial \phi^{m}}{\partial \phi^{\prime b}}+\frac{\partial^{2} \phi^{i}}{\partial \phi^{r} \partial \phi^{\prime b}} \frac{\partial \phi^{\prime a}}{\partial \phi^{i}}\right)
$$

Usually people associate a connection with a space endowed with a metric but this is not a necessary condition. In fact connection can also be built [7] out of a space equipped only with a symplectic structure $\omega$ as our space $\mathcal{M}$ is. The difference with metric spaces is that, while there the requirement that the connection preserve the metric scalar product between vectors plus that it is torsionless makes the connection unique, in symplectic spaces the analogue requirement that the connection preserves the symplectic scalar product ${ }^{2}$ does not make the connection unique [0]. Even if not unique what is important is that "there is a symplectic connection on any symplectic manifold" (see Proposition (2.5.2) on pag.66 of ref. [7]). Few more details are contained in the appendix.

Using a symplectic connection we can now build the following object which is a generalization of the variable $\lambda$ :

$$
\Lambda_{a} \equiv \lambda_{a}+i c^{l} \Gamma_{a l}^{m} \bar{c}_{m}
$$

and we can prove (see the calculations in the appendix ) that $\Lambda$, differently than $\lambda$ (see eq.(21)), transforms as a vector under a diffeomorphism generated by a vector field $\varepsilon^{a}(\phi)$, i.e:

$$
\Lambda_{a}^{\prime}=\Lambda_{a}+\Lambda_{b} \partial_{a} \epsilon^{b}
$$

One more thing that we would like to study is the manner to rewrite the Liederivative $\widetilde{\mathcal{H}}_{V}$ (14) using the variables $\Lambda_{a}$. We will restrict ourselves to Lie-derivatives of an Hamiltonian flow where $V^{a} \equiv \omega^{a b} \partial_{b} H$ with $H$ a function in phase-space but not necessarily the Hamiltonian of the system. The reason we want to rewrite $\widetilde{\mathcal{H}}_{V}$ using $\Lambda$ is because $\widetilde{\mathcal{H}}_{V}$ was a scalar despite the fact that it was built out of objects like $\lambda_{a}$ which did not have a tensorial character. It seems more natural to write $\widetilde{\mathcal{H}}_{V}$ out of objects which have a clear tensorial character as $\Lambda$. Let us first rewrite $\widetilde{\mathcal{H}}_{V}$ of eq. (14) in the following way:

$$
\widetilde{\mathcal{H}}_{V} \equiv \lambda_{a} V^{a}-i c^{b} \partial_{b} V^{a} \bar{c}_{a}
$$

Here we have just exchanged $c$ with $\bar{c}$ with respect to eq.(14). The difference between this $\widetilde{\mathcal{H}}_{V}$ and the one of eq.(14) is zero because the extra piece which would be produced is $\partial_{a} V^{a}$ which, with $V^{a}=\omega^{a b} \partial_{b} H$, is zero. This extra piece would be produced if we think of the $c$ and $\bar{c}$ as acting inside the path-integral and so having an anticommutator different from zero. Let us now replace the $\lambda_{a}$ in (42) with the $\Lambda_{a}$ using the relation

\footnotetext{
${ }^{12}$ By symplectic scalar product between two vectors $V^{a}$ and $W^{b}$ we mean: $V W \equiv V^{a} \omega_{a b} W^{b}$.
} 
(40). We obtain:

$$
\begin{aligned}
\widetilde{\mathcal{H}}_{V} & =V^{a}\left[\Lambda_{a}-i c^{l} \Gamma_{a l}^{m} \bar{c}_{m}\right]-i c^{b} \partial_{b} V^{a} \bar{c}_{a} \\
& =V^{a} \Lambda_{a}-i c^{b}\left[\partial_{b} V^{a}+\Gamma_{m b}^{a} V^{m}\right] \bar{c}_{a} \\
& \equiv V^{a} \Lambda_{a}-i c^{b} \partial_{; b} V^{a} \bar{c}_{a}
\end{aligned}
$$

In the last line of the equation above we have introduced the covariant derivative on vectors $V^{a}$ which is defined [6] as $\partial_{; b} V^{a} \equiv \partial_{b} V^{a}+\Gamma_{m b}^{a} V^{m}$. The covariant derivative has the well-known [6] property that $\partial_{; b} V^{a}$ transform as a tensor under diffeomorphism of $\phi$ while the usual derivative $\partial_{b} V^{a}$ does not. Looking at eq.(43) we see that $\widetilde{\mathcal{H}}_{V}$ has the same form as the $\widetilde{\mathcal{H}}_{V}$ of eq.(42) but with the replacements:

$$
\begin{aligned}
& \lambda_{a} \Longrightarrow \Lambda_{a} \\
& \partial_{a} \Longrightarrow \partial_{; a}
\end{aligned}
$$

These substitutions replace non-covariant expressions with covariant ones. As the $\widetilde{\mathcal{H}}_{V}$ was a covariant quantity (a scalar), it was natural to expect that it would not change at all under this replacement and in fact the $\widetilde{\mathcal{H}}_{V}$ in eq.(43) is the same, even if written with covariant quantities, as the one in (42).

Using this analysis we could ask the question of when is that an observable $\mathcal{O}(\phi, \lambda, c, \bar{c})$ is a scalar. The answer is the following: take $\mathcal{O}$ and use the relation (40) to replace $\lambda$ with $\Lambda$. If what you get has the same functional form in $\Lambda$ as $\mathcal{O}$ had in $\lambda$, with at most the ordinary derivatives replaced by covariant derivatives, then $\mathcal{O}$ is a scalar.

Let us now turn to the initial issue of building symmetric tensors. This is something we failed in doing [2] by using only $\lambda$. The only thing we succeeded was in building [1] anti-symmetric tensors using the $\bar{c}$. Let us note that, besides transforming correctly as vectors, the $\Lambda_{a}$ also commute among themselves. Thanks to these properties we have a manner to build symmetric tensors as

$$
\mathcal{T} \equiv \mathcal{T}^{a b \cdots n} \Lambda_{a} \Lambda_{b} \cdots \Lambda_{n}
$$

This ${ }^{[3]}$ settles one of the points which was not clear in [1]. What seems really impossible in our formalism is a manner to build mixed tensors. The reader may be tempted to build strings of objects containing both $\Lambda$ and $\bar{c}$ like $\mathcal{P}=\mathcal{P}^{a b c \cdots l m n} \Lambda_{a} \Lambda_{b} \Lambda_{c} \cdots \bar{c}_{l} \bar{c}_{m} \bar{c}_{n}$, but this is anti-symmetric in the exchange of the $\bar{c}$ among themselves and symmetric in the exchange the $\Lambda$ among themselves and with the $\bar{c}$ so it is not a mixed tensor.

\footnotetext{
${ }^{13}$ The reader should remember that here we mean "commuting" among the $\Lambda$ not in an operatorial sense but in the sense of considering $c$ and $\bar{c}$ entering the $\Lambda$ as Grassmannian variables which anticommutes among themselves. The $\bar{c}$ acquires the operatorial meaning of being the $\frac{\partial}{\partial c}$ only once it is inserted into the path-integral. Here,instead, in building the tensors $\mathcal{T}$ of eq.(46) we do not need to use the path-integral at all, that is why the $\Lambda$ "commutes" among themselves.
} 


\section{Geometric Structure.}

In this section we will try to understand which kind of space is the one labeled by our 8n coordinates $(\phi, \lambda, c, \bar{c})$ and correct some wrong statements present in [1] [2].

As we said at the end of section 2 the base-space to be considered is the one labeled by $(\phi, c)$. Let us then first find out which kind of space is this. $\phi^{a}$ are the $2 \mathrm{n}$ coordinates of the phase-space $\mathcal{M}$. The $c^{a}$ transform under a diffeomorphism (see eq.(16)) as the forms $d \phi^{a}$. So we stated in refs. [1] [2] that, identifying $c$ with $d \phi$, the space $\left(\phi^{a}, c^{a}\right)$ makes up the cotangent bundle [4] to phase space: $T^{\star} \mathcal{M}$. That is wrong!. In fact $c^{a}$ are at most a basis in the fiber $T_{\phi}^{\star} \mathcal{M}$ and not a generic vector in $T_{\phi}^{\star} \mathcal{M}$. Being the $c$ a basis they belong to what is called [8] the bundle of linear frames to $\mathrm{M}$. So the $\left(\phi^{a}, c^{a}\right)$ are nothing else than a section of the linear-frame-bundlem. We say a section because there are other basis (or frames), besides $c^{a}$, which one could choose in the fibers of the linear-frame-bundle.

As we stressed the structure above holds if one identifies $c^{a}$ with the $d \phi^{a}$. We did that identification in ref. [1] and used it to turn the whole Cartan calculus into operations which could be done via our path-integral and the structures (commutators, BRS charges, etc) present in it. Of course the fact that the $c^{a}$ transforms as the $d \phi^{a}$ does not force us to identify them so explicitly as we have done in ref. [1]. For example if we build a generic vector field $V \equiv V^{a} \frac{\partial}{\partial \phi^{a}}$, we would have that the components $V^{a}$ transforms as the $c^{a}$, so we could do this identification and the $c^{a}$ would then be components (and not basis) of the vectors in the tangent fibers and they would make up with the $\phi$ the tangent bundle to phase-space $T \mathcal{M}$. The problem is that the $c^{a}$ have a Grassmannian nature and not like the $V^{a}$ a bosonic one. In that case the bundle is called [3] reversed parity tangent bundle and indicated as $\Pi T \mathcal{M}$.

Next we have to consider the role of the $4 \mathrm{n}$ remaining variables $\left(\lambda_{a}, \bar{c}_{a}\right)$. Looking at the Lagrangian in (1) we see that they play the role of momenta to the variables $\left(\phi^{a}, c^{a}\right)$, so they will make the cotangent fibers to the previous space. We can summarize all this in the following scheme:

$$
\begin{aligned}
\left(\phi^{a}\right) & \Longrightarrow \mathcal{M} \\
\left(\phi^{a}, c^{a}\right) & \Longrightarrow \Pi T \mathcal{M} \\
\left(\phi^{a}, c^{a}, \lambda_{a}, \bar{c}_{a}\right) & \Longrightarrow T^{\star}(\Pi T \mathcal{M})
\end{aligned}
$$

On the other hand in ref. [1] (eq.3.33) we proved that the $\bar{c}_{a}$ act, in the operatorial counterpart of our path-integral, as $\bar{c}_{a}=\frac{\partial}{\partial c^{a}}$. Moreover in the previous section of

\footnotetext{
${ }^{14}$ Actually the bundle-of-linear-frames is made out of basis of the tangent fibers while ours is of cotangent fibers, but the two are isomorphic.

${ }^{15}$ That way to look at our variables is the one we basically adopted, without realizing it, in refs. [1] [2] and in the previous three sections of this paper.
} 
this paper we proved that $\lambda_{a}$, despite their strange transformation properties (6), still maintain their operatorial meaning of being: $\lambda_{a}=-i \frac{\partial}{\partial \phi^{a}}$. From these two expressions we can say that $\left(\lambda_{a}, \bar{c}_{a}\right)$ form a basis in the tangent fibers to the base-space $\left(\phi^{a}, c^{a}\right)$. As this base-space was $\Pi T \mathcal{M}$, the over-all $8 \mathrm{n}$ coordinates $\left(\phi^{a}, c^{a}, \lambda_{a}, \bar{c}_{a}\right)$ can also be considered as a section of the bundle of linear frames over $\Pi T \mathcal{M}$. This is an alternative interpretation of our $8 \mathrm{n}$ variables with respect to the interpretation contained in eq. (49). This sort of "duality" between considering each of our variables $(\lambda, c, \bar{c})$ either as basis or as coordinate could be considered at each of the levels of eqs.(48)(49) and gives rise to all possible combinations. We will anyhow stick here to the "coordinate"-picture which will lead to the "reversed-parity" bundle of eq.(49).

Beside this sort of "duality" which would allow us to see in two different ways the spaces labeled by our variables, there is a further freedom. This is related to the scheme of eqs.(48)(49). Let us perform a partial integration in the kinetic piece of the Grassmannian variables present in the Lagrangian (1). Modulo surface terms the new Lagrangian is

$$
\mathcal{L}^{\prime} \equiv \lambda_{a}\left[\dot{\phi}^{a}-\omega^{a b} \partial_{b} H\right]-i \dot{\bar{c}}_{a} c^{a}-i \bar{c}_{a} \omega^{a c} \partial_{c} \partial_{b} H c^{b}
$$

Being this Lagrangian different from $\widetilde{\mathcal{L}}$ only by a surface term, the equations of motion for $c^{a}$ and $\bar{c}_{a}$ are the same, but now $\bar{c}_{a}$ plays the role of a "configurational" variable while $c^{a}$ is its relative momentum. Then it would seem natural to choose in eq. (48) as new variables $\left(\phi^{a}, \bar{c}_{a}\right)$. Let us see its geometrical interpretation: the $\bar{c}_{a}$ transform as (17) so, interpreting the $\bar{c}_{a}$ as "coordinates" and not "basis", they transform as components of forms but with Grassmannian character, i.e. with the reversed parity character. This means that the $\left(\phi^{a}, \bar{c}_{a}\right)$ are the coordinates of the reversed parity cotangent bundle:

$$
\left(\phi^{a}, \bar{c}_{a}\right) \Longrightarrow \Pi T^{\star} M
$$

From the Lagrangian $\mathcal{L}^{\prime}$ of (50) we see that $\lambda_{a}$ and $c^{a}$ play the role of momenta to the previus variables so they belong to the cotangent fibers of the previous space. All together then we can write all this in the following scheme

$$
\begin{aligned}
\left(\phi^{a}\right) & \Longrightarrow \mathcal{M} \\
\left(\phi^{a}, \bar{c}_{a}\right) & \Longrightarrow \Pi T^{\star} \mathcal{M} \\
\left(\phi^{a}, \bar{c}_{a}, \lambda_{a}, c^{a}\right) & \Longrightarrow T^{\star}\left(\Pi T^{\star} \mathcal{M}\right)
\end{aligned}
$$

As the physics contained in the Lagrangians $\mathcal{L}$ and $\mathcal{L}^{\prime}$ is the same and the same are the coordinates, we could say that our variables label both spaces either $T^{\star}(\Pi T \mathcal{M})$ or $T^{\star}\left(\Pi T^{\star} \mathcal{M}\right)$. A more mathematically precise proof of this is contained in ref. [10. 


\section{$5 \quad$ Entirely Bosonic Path Integral.}

The reader may feel a little been uneasy with these Grassmannian double bundle we have provided in the previous section and even with the alternative interpretation as sections of the frame bundle. For this reason in this section we will show that, at least for Hamiltonian flow, it is possible to provide a path-integral of classical mechanics made entirely of bosonic variables. Moreover we will prove that these variables are just the coordinates of a standard double bundle like $T^{\star}\left(T^{\star} \mathcal{M}\right)$. The procedure to achieve what we said above is explained below.

The path-integral [1] for classical mechanics (CM) was basically the following. We wanted to create a generating functional $Z[J]$ which would give to each path not the Feynman weight $\exp \frac{i}{\hbar} S$ but weight 1 if the path was a classical one and weight zero if it was a non-classical one. So essentially:

$$
Z[J]=\int \mathcal{D} \phi \tilde{\delta}\left[\phi(t)-\phi_{c l}(t)\right] \exp \int J \phi d t
$$

where $\phi_{c l}$ are the solutions of the Hamiltonian equations of motion: $\dot{\phi}^{a}=\omega^{a b} \frac{\partial H}{\partial \phi^{b}}$ and the $\tilde{\delta}[\cdot]$ is a functional Dirac delta. It is easy to realize [1], neglecting the current $J$ for a moment, we can rewrite $(55)$ as

$$
\begin{aligned}
Z[J]=\int \mathcal{D} \phi \tilde{\delta}\left[\phi(t)-\phi_{c l}(t)\right] & =\int \mathcal{D} \phi \tilde{\delta}\left[\dot{\phi}^{a}-\omega^{a b} \frac{\partial H}{\partial \phi^{b}}\right] \operatorname{det}\left[\delta_{l}^{a} \partial_{t}-\omega^{a b} \frac{\partial^{2} H}{\partial \phi^{b} \partial \phi^{l}}\right]( \\
& =\int \mathcal{D} \phi^{a} \mathcal{D} \lambda_{a} \mathcal{D} c^{a} \mathcal{D} \bar{c}_{a} \exp i \int \tilde{\mathcal{L}} d t
\end{aligned}
$$

where the $\operatorname{det}[\cdot]$ appearing in eq.(56) is a functional determinant 11 and the $\widetilde{\mathcal{L}}$ in (57) is the Lagrangian of eq.(11). It is obtained by doing the Fourier transform (via the variables $\lambda_{a}$ ) of the Dirac delta in the second term of eq.(56) and exponentiating the $\operatorname{det}[\cdot]$ with Grassmannian variables $c^{a}$ and $\bar{c}_{a}$. In order to avoid using the Grassmannian variables the trick we adopt now is to substitute the $\operatorname{det}[\cdot]$ in (56) with an inverse determinant:

$$
\operatorname{det}\left[\delta_{l}^{a} \partial_{t}-\omega^{a b} \frac{\partial^{2} H}{\partial \phi^{b} \partial \phi^{l}}\right]=\left\{\operatorname{det}\left[\delta_{l}^{a} \partial_{t}+\omega^{a b} \frac{\partial^{2} H}{\partial \phi^{b} \partial \phi^{l}}\right]\right\}^{-1}
$$

We will give a detailed proof of this relation in the appendix.

The next step is to use relation (58) into (56), then "exponentiate" the inverse of the matrix using bosonic variables by making use of the well known formula 1 t:

$$
\int d x^{i} d y_{j} \exp i x^{i} A_{i}^{j} y_{j} \propto\left\{\operatorname{det}\left[A_{i}^{j}\right]\right\}^{-1}
$$

\footnotetext{
${ }^{16}$ This formulas requires that the determinant be positive and this is our case because the LHS of (58) is positive [1].
} 
Doing all that we get

$$
\begin{aligned}
Z[J] & =\int \mathcal{D} \phi \tilde{\delta}\left[\dot{\phi}^{a}-\omega^{a b} \frac{\partial H}{\partial \phi^{b}}\right]\left\{\operatorname{det}\left[\delta_{l}^{a} \partial_{t}+\omega^{a b} \frac{\partial^{2} H}{\partial \phi^{b} \partial \phi^{l}}\right]\right\}^{-1} \\
& =\int \mathcal{D} \phi^{a} \mathcal{D} \lambda_{a} \mathcal{D} \pi^{a} \mathcal{D} \xi_{a} \exp i \int \mathcal{L} d t
\end{aligned}
$$

where

$$
\mathcal{L}=\lambda_{a}\left[\dot{\phi}^{a}-\omega^{a b} \frac{\partial H}{\partial \phi^{b}}\right]+\pi^{l}\left[\delta_{l}^{a} \partial_{t}+\omega^{a b} \frac{\partial^{2} H}{\partial \phi^{b} \partial \phi^{l}}\right] \xi_{a}
$$

The variables $\pi^{l}$ and $\xi_{a}$ are the Bosonic variables we have used to exponentiate the inverse matrix and they replace the Grassmannian variables $c^{a}$ and $\bar{c}_{a}$ present in $\widetilde{\mathcal{L}}$ of eq.(11).

Let us now see if we can give a geometrical understanding of the new variables $\pi^{a}, \xi_{a}$ present here. Let us show how they change under the Hamiltonian flow, that means under their equation of motion which can easily be derived from the Lagrangian $\mathcal{L}$ above:

$$
\partial_{t} \xi_{l}+\xi_{a} \omega^{a b} \frac{\partial^{2} H}{\partial \phi^{b} \partial^{l}}=0
$$

This equation should be compared with the equations of motion of $c^{a}$ derived [1] from $\widetilde{\mathcal{L}}$ of eq.(四) which are

$$
\partial_{t} c^{a}-\omega^{a b} \frac{\partial^{2} H}{\partial \phi^{b} \partial \phi^{l}} c^{l}=0
$$

From the above equations it is now easy to see that the quantity

$$
\Xi \equiv \xi_{a} c^{a}
$$

is invariant under the Hamiltonian flow. This quantity would behave in the same way under any diffeomorphism of the phase-space $\mathcal{M}$ and not just under the Hamiltonian flow

$$
\tilde{\Xi} \equiv \tilde{\xi}_{a} d \phi^{a}
$$

and by identifying the $d \phi^{a}$ above with the $c^{a}$ of eq.(65) we see that we can identify $\xi_{a}$ of (65) with the components $\tilde{\xi}_{a}$ of the forms of (66). So while the $c^{a}$ are the basis of the fibers on $T^{\star} \mathcal{M}$, the $\xi_{a}$ are the coordinates of the same space. Looking at the Lagrangian (62) we see that $\pi^{a}$ and $\lambda_{a}$ are the momenta associated to $\phi^{a}$ and $\xi_{a}$, that means they will make up the cotangent fibers to the previous space, So the overall set of variables $\left(\phi^{a}, \xi_{a}, \lambda_{a}, \pi^{a},\right)$ are the coordinates of $T^{\star}\left(T^{\star} \mathcal{M}\right)$. This is a double bundle

\footnotetext{
${ }^{17}$ This is so because we would have to choose the transformations on $\pi$ and $\xi$ induced by the diffeomorphism in $\phi$ in such a way to keep invariant the Hamiltonian associated to $\mathcal{L}$.

18 This is so because forms are object totally coordinate free.
} 
but it may please more the reader that the reversed parity one $T^{\star}(\Pi T \mathcal{M})$ associated to the Lagrangian $\tilde{\mathcal{L}}$ of (1). It may be a space easier to handle for the study of various physical issues like the study of ergodicity and Lyapunov exponents [9] we performed previously using the old Lagrangian (1). It may also be worth to see if the universal symmetries (BRS and Supersymmetry) we found in [1] [9] are present (in a different form) also in this purely bosonic case presented here.

\section{Conclusions}

In this paper we have settled several geometrical issues still opened in the path-integral approach to classical mechanics. We think that the light shed on the "dual" aspects of the geometrical interpretations of the Grassmannian variables is something important and long overdue. At the same time we have disentangled the puzzles related to the $\lambda$ variables understanding the manner to build symmetric tensors. Last but not least we have to say that having cleared all the geometry involved has helped us in our search for a purely bosonic path-integral. Overall we think that it was not useless to do all this work especially considering the relevant role that geometry is having in physics in the last ten years. 


\section{Appendix}

In this appendix we provide some calculational details which will make the paper selfcontained.

- As we said in section 3 there are various extra conditions which one could impose on the symplectic connections. One condition, which anyhow will not make the connection unique, is the requirement that the connection be torsionless which is equivalent to saying that it must be symmetric in the two lower indices [6]: $\Gamma_{a b}^{c}=\Gamma_{b a}^{c}$. For the reader not familiar with torsion we can phrase this requirement in the following other form: having a connection one can build a covariant derivative on the space of forms. It is easy to see that, if we write forms as $\mathcal{F}=\mathcal{F}_{a b \cdots m} c^{a} c^{b} \cdots c^{m}$, the covariant derivative acting on them is

$$
\nabla_{a} \equiv \frac{\partial}{\partial \phi^{a}}-c^{l} \Gamma_{a l}^{m} \frac{\partial}{\partial c^{m}}
$$

Using it we could then obtain a new" exterior derivative". The old one was [四] $d=c^{a} \frac{\partial}{\partial \phi^{a}}$ and the new one would be $\tilde{d} \equiv c^{a} \nabla_{a}=c^{a}\left[\frac{\partial}{\partial \phi^{a}}-c^{l} \Gamma_{a l}^{m} \frac{\partial}{\partial c^{m}}\right]$. Here one see immediately that if $\Gamma$ were symmetric in the two lower indeces, the $\tilde{d}$ would turn into the old exterior derivative $d$. So requiring this symmetry in the lower indices would not only make our theory torsionless but it would also prevent the appearance of two different exterior derivatives.

- As we promised after eq.(40) we will show here that $\Lambda$ transforms as a vector under a diffeomorphism of the phase-space : $\phi^{a} \rightarrow \phi^{a}(\phi)$. Let us first write $\Lambda$ as $\Lambda_{a}=\lambda_{a}+i W_{a}$ where (see eq.(40) $W_{a} \equiv c^{l} \Gamma_{a l}^{m} \bar{c}_{m}$. We know how $\lambda$ transforms under the diffeomorphism indicated above (see eq.(29)) and so we have only to find how

$W$ changes under the same transformations. Using the transformations rules for $\Gamma$ (eq.(39)) and for $c$ and $\bar{c}$ which are:

$$
\begin{aligned}
c^{\prime a} & =\frac{\partial \phi^{\prime a}}{\partial \phi^{b}} c^{b} \\
\bar{c}_{a}^{\prime} & =\frac{\partial \phi^{b}}{\partial \phi^{\prime a}} \bar{c}_{b}
\end{aligned}
$$

It is a simple but long calculation to show how the $W_{a}$ transform:

$$
\begin{aligned}
W_{a}^{\prime} & =\frac{\partial \phi^{r}}{\partial \phi^{\prime a}} W_{r}+\frac{\partial^{2} \phi^{j}}{\partial \phi^{\prime a} \partial \phi^{\prime l}} \frac{\partial \phi^{\prime l}}{\partial \phi^{k}} c^{k} \bar{c}_{j} \\
& =\frac{\partial \phi^{r}}{\partial \phi^{\prime a}} W_{r}+\frac{\partial c^{s}}{\partial \phi^{\prime a}} \bar{c}_{s}
\end{aligned}
$$

Combining the equation above with (29) we get that: 


$$
\begin{aligned}
\Lambda_{a}^{\prime} & \equiv \lambda_{a}^{\prime}+i W_{a}^{\prime} \\
& =\frac{\partial \phi^{b}}{\partial \phi^{\prime a}} \lambda_{b}-i \frac{\partial c^{b}}{\partial \phi^{\prime a}} \bar{c}_{b}+i \frac{\partial \phi^{b}}{\partial \phi^{\prime a}} W_{b}+i \frac{\partial c^{b}}{\partial \phi^{\prime a}} \bar{c}_{b} \\
& =\frac{\partial \phi^{b}}{\partial \phi^{\prime a}} \lambda_{b}+i \frac{\partial \phi^{b}}{\partial \phi^{\prime a}} W_{b}=\frac{\partial \phi^{b}}{\partial \phi^{\prime a}} \Lambda_{b}
\end{aligned}
$$

If the transformation is generated by a vector field $\varepsilon^{a}(\phi)$, i.e.: $\phi^{\prime a}=\phi^{a}-\varepsilon^{a}(\phi)$, then eq.(71) becomes exactly eq.(41). Eq.(71) proves that $\Lambda$ transforms as a vector. Somehow the extra pieces in the transformations of $\lambda$ (eq.(29)) got cancelled by the extra pieces in the transformations of $W$ (in eq. (70)).

- We will now give a formal proof of formula (58). The determinant in (58) are functional determinant that means:

$$
\begin{aligned}
\operatorname{det}\left[\delta_{l}^{a} \partial_{t}-\omega^{a b} \frac{\partial^{2} H}{\partial \phi^{b} \partial \phi^{l}}\right] & \equiv \operatorname{det}\left[\delta_{l}^{a} \partial_{t} \delta\left(t-t^{\prime}\right)-\delta\left(t-t^{\prime}\right) \omega^{a b} \frac{\partial^{2} H}{\partial \phi^{b} \partial \phi^{l}}\right] \\
& =\left\{\operatorname{det} \partial_{t}\right\}\left\{\operatorname{det}\left[\delta_{l}^{a} \delta\left(t-t^{\prime}\right)-\theta\left(t-t^{\prime}\right) \omega^{a b} \frac{\partial^{2} H}{\partial \phi^{b} \partial \phi^{l}}\right]\right\}
\end{aligned}
$$

To prove (58) is equivalent to saying that the determinant of the product of the two matrices appearing respectively on the LHS and RHS of (58) is one. Using the form of the matrix written in eq. (73), this meanst5:

$$
\begin{aligned}
& \operatorname{det}\left\{\int d t^{\prime}\left[\delta_{b}^{a} \delta\left(t-t^{\prime}\right)-\theta\left(t-t^{\prime}\right) \omega^{a l} \frac{\partial^{2} H}{\partial \phi^{l} \partial \phi^{b}}\right]\left[\delta_{c}^{b} \delta\left(t^{\prime}-t^{\prime \prime}\right)+\theta\left(t^{\prime}-t^{\prime \prime}\right) \omega^{b k} \frac{\partial^{2} H}{\partial \phi^{k} \partial \phi^{c}}\right]\right\}= \\
& \operatorname{det}\left\{\delta_{c}^{a} \delta\left(t-t^{\prime \prime}\right)-\int d t^{\prime} \theta\left(t-t^{\prime}\right) \theta\left(t^{\prime}-t^{\prime \prime}\right) \omega^{a l} \frac{\partial^{2} H}{\partial \phi^{l} \partial \phi^{b}} \omega^{b k} \frac{\partial^{2} H}{\partial \phi^{k} \partial \phi^{c}}\right\} \\
& \approx \quad \exp -\int d t^{\prime} \theta\left(t-t^{\prime}\right) \theta\left(t^{\prime}-t\right) \omega^{a l} \frac{\partial^{2} H}{\partial \phi^{l} \partial \phi^{b}} \omega^{b k} \frac{\partial^{2} H}{\partial \phi^{k} \partial \phi^{a}}=1
\end{aligned}
$$

In (75) we have used the "exp-tr" form for the determinant and fact that the two $\theta(\cdot)$ give zero. So this proves the relation (58).

- Here we will qualify the steps done in eqs.(74) and (75). There we used the $\theta\left(t-t^{\prime}\right)$ as "inverse" (or Green function) of $\partial_{t}$. The $\theta\left(t-t^{\prime}\right)$ is the retarted or causal Green function. If we had used other Green functions like the $\epsilon\left(t-t^{\prime}\right)$, we would not have obtained equation (75).

It is actually well-known [1] 12 that all functional determinants of the form:

$$
\operatorname{det}\left[\partial_{t} \delta\left(t-t^{\prime}\right)-\delta\left(t-t^{\prime}\right) G^{\prime}(\varphi)\right]
$$

\footnotetext{
${ }^{19}$ We can use that form of the matrix because they differ by a constant factor independent of the $\phi$.
} 
depend on the boundary conditions under which we solve the associated differential equation:

$$
\left[\partial_{t}-G^{\prime}(\varphi)\right] c_{n}(t)=\sigma_{n} c_{n}(t)
$$

whose eigenvalues $\sigma_{n}$ are needed to calculate the determinant in some regularized form:

$$
\operatorname{det}[(\cdot)]=\left\{\prod_{n=-\infty}^{n=\infty} \sigma_{n}\right\}_{\text {regul }}
$$

Solving equation (77) with causal boundary conditions one obtains [12]:

$$
\operatorname{det}\left[\partial_{t} \delta\left(t-t^{\prime}\right)-\delta\left(t-t^{\prime}\right) G^{\prime}(\varphi)\right]_{\text {causal. }}=\exp -\frac{1}{2} \int d t^{\prime} G^{\prime}\left(\varphi\left(t^{\prime}\right)\right)
$$

So by reversing the sign of $G^{\prime}(\varphi)$ we get:

$$
\operatorname{det}\left[\partial_{t} \delta\left(t-t^{\prime}\right)+\delta\left(t-t^{\prime}\right) G^{\prime}(\varphi)\right]_{\text {causal. }}=\exp +\frac{1}{2} \int d t^{\prime} G^{\prime}\left(\varphi\left(t^{\prime}\right)\right)
$$

By comparing the RHS of the last two equations above we see that the two determinants are one the inverse of the other. This proves relation (58) provided we specify that the functional determinant has to be evaluated with causal boundary conditions.

The reason we choose these boundary conditions is because, after all, we are just doing classical mechanics that means just solving ordinary Hamiltonian equations of motion. These are usually solved by giving a value of $\varphi$ at the intial time $t=0$ and looking for the evolution at later times using a causal propagator. The use of periodic b.c. and of a time-symmetric Green function for our path-integral has been analyzed in full details in ref. [13. The result is a path-integral whose only non-zero expectation values are those associated to observables which are independent from deformations of the Hamiltonian $H$ and of its symplectic form $\omega_{a b}$. This means a path-integral which

does not feel anymore the form of $\mathrm{H}$, so something that does not feel the dynamics at all and this is is not what we want here.

\section{Acknowledgments}

We are expecially grateful to D.Mauro for many discussions and much needed help during the last stages of this work. We are also grateful to G.Landi, G.Marmo for helpful discussions and to F.Benatti who, since December 1994, insisted that we find a purely bosonic representation of our path-integral. This work has been supported by grants from MURST (Italy) and NATO. 


\section{References}

[1] E.Gozzi, M.Reuter and W.D.Thacker, Phys.Rev.D 40, 3363 (1989).

[2] E.Gozzi, M.Reuter and W.D.Thacker, Phys. Rev.D 46, 757 (1992).

[3] A.Schwarz, in "Topics in statistical and theoretical physics" Ed. R.L.Dobrushin.

[4] R.Abraham and J.Marsden, "Foundations of Mechanics" (Benjamin, New York, 1978).

[5] I.Kolar, P.W.Michor, J.Slovak, "Natural Operations in Differential Geometry" (Springer Verlag 1993, Berlin).

[6] S.P.Novikov, B.A.Dubrovin, A.T.Fomenko, "Modern geometric methods and applications." Springer-Verlag, New York, 1992.

[7] B.Fedosov, "Deformation quantization and index theory" Akademie Verlag, Berlin 1996.

[8] S.Kobayashi and K.Nomizu, "Foundations of differential geometry" Vol.I John Wiley and Sons Editors, New York 1963

[9] E.Gozzi and M.Reuter, Phys. Lett. B 233 (1989) 383; Chaos, Solitons and Fractals, Vol.4, no.7 (1994) 1117.

[10] Marco Regini, Tesi di Laurea, Universita' di Trieste, July 1998.

[11] H.Nakazato et al, Nucl.Phys. B346 (1990) 611-631;

[12] S.Marculescu et al., Nucl.Phys. B 349 (1991) 463-493;

[13] E.Gozzi, M.Reuter, Phys. Lett. B 240 (1990) 137. 